\title{
CLDN2 inhibits the metastasis of osteosarcoma cells via down-regulating the afadin/ERK signaling pathway
}

\author{
Xiaowei Zhang ${ }^{1,3}$, Haiming Wang ${ }^{2}$, Qian $\mathrm{Li}^{3}$ and Tao $\mathrm{Li}^{1,3^{*}}$
}

\begin{abstract}
Background: In an earlier study, we investigated the expression of tight junction protein claudins (CLDNs) in human osteosarcoma (OS) cells, and the CLDN2 was found to be down-regulated in primary tumor cells compared with normal osteoblast cells. Here, we sought to explore the effects of CLDN2 on the malignant phenotype of OS and the underlying molecular mechanisms.

Methods: The expression patterns of CLDN2 and afadin in OS tissues and histologically non-neoplastic bone tissues were explored via immunohistochemistry and western blotting. CLDN2 expression levels in an OS cell line stably expressing CLDN2 and an osteoblast cell line with a CLDN2 knockout were confirmed by western blotting and immunofluorescence staining. The malignant phenotype of OS cells and osteoblast cells in vitro was assessed using a cell counting kit-8 assay, transwell assay and wound-healing experiment. Western blotting was utilized to detect the activation state of Ras/Raf/MEK/ERK pathway. Moreover, an RNA interference method were used to silence afadin in CLDN2-expressing OS cells.
\end{abstract}

Results: Our research group found that CLDN2 and afadin was underexpressed in OS tissues, and the overexpression of CLDN2 significantly inhibited the migration abilities of OS cells. Genetic silencing of afadin in CLDN2-overexpressing OS cells promoted U2OS cell motility and activation of the Ras/Raf/MEK/ERK pathway.

Conclusions: In this study, we confirmed that CLDN2 expression significantly inhibited the malignant phenotype of OS cells in vitro. Inhibition of the ERK pathway by afadin may be one of the mechanisms by which CLDN2 blocks the metastasis phenotype of OS cells.

Keywords: Afadin, Osteosarcoma, Claudin-2, Extracellular signal-regulated kinase, Metastasis, Tight junction

\section{Background}

The main reason for the poor prognosis of osteosarcoma (OS) is recurrence and metastasis, and the 5-year survival rate after recurrence and metastasis is less than $30 \%[1,2]$. At present, the issue of controlling the early metastasis of OS has become a bottleneck in the treatment of this disease [3]. Previous studies have shown that for various human malignancies, metastasis is accompanied by abnormalities in tight junction (TJ)

\footnotetext{
*Correspondence: taolichina@126.com

${ }^{1}$ Center for Translational Medicine, Central Hospital of Zibo, Affiliated with Shandong University, Gong qingtuan Road 54Hao, Zibo, Shandong, China

Full list of author information is available at the end of the article
}

structure and function $[4,5]$. TJs are located at the very apex of cell junctions and both regulate cell adhesion and maintain cell polarity and permeability [6]. Recent research has shown that in addition to their functions in the maintenance of cell polarity and permeability, TJs are also reportedly involved in the transduction of intracellular/extracellular signals and the regulation of cell proliferation, differentiation, invasion and migration $[7,8]$. Claudins (CLDNs) are the structural proteins that form TJs, and abnormalities in tight junction structure and function caused by a lack of CLDN expression on the cell membrane are required for tumor cell invasion and metastasis $[9,10]$. Moreover, a growing number of studies in recent years have revealed that tumor cells

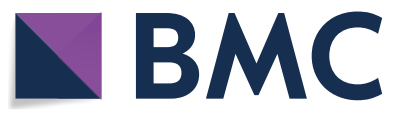

(c) The Author(s) 2018. This article is distributed under the terms of the Creative Commons Attribution 4.0 International License (http://creativecommons.org/licenses/by/4.0/), which permits unrestricted use, distribution, and reproduction in any medium, provided you give appropriate credit to the original author(s) and the source, provide a link to the Creative Commons license, and indicate if changes were made. The Creative Commons Public Domain Dedication waiver (http://creativecommons.org/ publicdomain/zero/1.0/) applies to the data made available in this article, unless otherwise stated. 
frequently exhibit changes in the expression and localization of CLDNs [11]. For instance, it has been reported that CLDN1 is a metastasis suppressor for lung adenocarcinoma and that a lack of CLDN1 expression is associated with poor patient prognosis [12]. In addition, studies by Usami et al. indicated that reduced CLDN7 expression in head and neck cancer cells promoted cell invasion and migration [13]. These studies indicate that specific expression patterns of CLDNs in tumors have the potential to be used as molecular markers of malignancies.

Accumulating evidence suggests that the carboxyl termini of CLDNs, which are located in the cytoplasm, participate in signal transduction through their PDZ (PSD-95/Dlg/ZO-1 homology domain)-binding domains, which bind to cytoplasmic PDZ motif-containing proteins, including zonula occludens (ZOs) and afadin, among others [14-16]. The binding of CLDNs to these proteins constitutes a step in cellular signal transduction [17]. Of these binding partners, afadin is a core TJ protein that can bind to other TJ proteins in the cell membrane under certain physiological conditions and play an important role in maintaining TJ functions [18]. Furthermore, recent studies have shown that afadin can competitively inhibit the binding of rat sarcoma (Ras) protein to rapidly accelerated fibrosarcoma (Raf) protein, inhibiting the activation of mitogen-activated protein kinase kinase $1 / 2($ MEK1/2) and blocking activation of the extracellular signal-regulated kinase (ERK) signal pathway [19]. However, no prior reports have described either the role of CLDN2 on the malignancy phenotype of OS cells or whether the impact of CLDN2 is related to the afadin/ ERK signaling pathway. In this study, we used molecular biological and other techniques to study the role and mechanisms of CLDN2 in OS cell metastasis and to identify novel targets for OS treatment and the control of early metastasis.

\section{Methods}

\section{Cell culture}

The human embryonic kidney cells 293 (HEK293T) cells, the human OS cell lines, U2OS and Saos2, and human fetal-osteoblast cell line, hFOB.1.19 were used in this study were purchased from Shanghai Cell Bank of the Chinese Academy of Sciences. These cell lines were maintained in Dulbecco's modified Eagle's medium supplemented with $10 \%$ fetal bovine serum (FBS) in a humidified incubator containing $5 \% \mathrm{CO}_{2}$ at $37^{\circ} \mathrm{C}$.

\section{Plasmid construction and transfection}

The pNSE-IRES-EGFP1-C1/CLDN2 (NM_001171095) was constructed and amplified by KeyGEN BioTECH Company. Five micrograms of each plasmid were transfected into cells using SuperFect Transfection Reagent
(TaKaRa, Japan) in accordance with the protocol. G418 (Sigma, St. Louis, Missouri, USA)-resistant clones were expanded in culture as a monoclonal population. Cells transfected with an empty vector pNSE-IRES2-EGFP1$\mathrm{C} 1(+)$ were used as a vector control.

\section{Realtime reverse transcript-polymerase chain reaction (RT-PCR)}

Total RNA was extracted using a Perfect Pure RNA Cultured Cell Kit (ThermoFisher Scientific, Waltham, MA) in line with the manufacturer's protocol. Realtime PCR reactions were carried out as previously pronounced [20]. Primers pairs of CLDNs and glyceraldehyde phosphate dehydrogenase (GAPDH) were as follows: CLDN1 forward (5'-GCCACAGCAAGGTATGGT AAC-3') and reverse (5'-AGTAGGGCACCTCCCAGA AG-3'); CLDN2 forward (5'-TTCATCGGCAACAGC ATCG-3') and reverse (5'-GGTTATAGAAGTCCCGGA TGA-3'); CLDN3 forward (5'-AGTGCAAGGTGTACG ACTC- $\left.3^{\prime}\right)$ and reverse (5'-AGTCCCGGATAATGGTGT TG-3'); CLDN4 forward (5'-TTGTCACCTCGCAGA CCATC- $3^{\prime}$ ) and reverse (5'-GCAGCGAGTCGTACA CCTTG-3'); CLDN5 forward (5'-AACATCGTGACG GCGCAGACCA-3') and reverse (5'-TCAGAGCCAGCA CCGAGTCGTACA-3'); CLDN6 forward (5'-GGCAAC AGCATCGTCGTGG-3') and reverse (5'-GAAGTCCTG GATGATAGAGTGGGC-3'); CLDN7 forward $\left(5^{\prime}\right.$-TTT TCATCGTGGCAGGTCTT-3') and reverse (5'-GGC CAAACTCATACTTAATGTTGG-3'); CLDN8 forward (5'-TCTGCAGTAGGA CATAGAAACCCCTAA- $\left.{ }^{\prime}\right)$ and reverse (5'-CGTTTAGGGGTTTCTATGTCCTACTGC3'); CLDN9 forward (5'-CTAGCACTAGTTTCGAAA TGGCT TCGACCGGCTTAG- $\left.3^{\prime}\right)$ and reverse (5'-TCT CGAGCTAGTCGACTCACACGTAGTCCC TCTTGT C-3') and GAPDH forward (5'-AACGTGTCAGTCGTG GACCTG- $3^{\prime}$ ) and reverse ( $5^{\prime}$-AGTGGGTGTCGCTGTFGAAGT- $\left.3^{\prime}\right)$. The reverse transcript cDNA reaction products were subjected to quantitative real-time PCR using CTFX 96 Real-time system (Bio-Rad, Hercules, CA, USA) and SYBR green supermix (Bio-Rad, Hercules, CA, USA) according to the manufacturer's protocol.

\section{Materials}

Rabbit polyclonal antibodies against CLDN2 (ab107059) and anti-human $\beta$-actin (ab8226) were purchased from Abcam (Massachusetts, US). Rabbit anti-human phospho-ERK1/2 (\#9251), rabbit anti-human ERK1/2 (\#4695), rabbit anti-human ras (\#14429), rabbit antihuman raf-1 (\#2330), rabbit anti-human phosphoMEK1/2 (\#2338), rabbit anti-human MEK1/2(\#8727) and rabbit anti-human afadin (\#13531) were purchased from Cell Signaling Technology (Boston, USA) and a 
streptavidin-peroxidase immunohistochemistry reagent kit was purchased from Maixin Biology (Fujian, China).

\section{Western blotting}

The protein concentration of cell lysates was determined using a bicinchoninic acid (BCA) Protein Assay Kit (Pierce Chemical Co., Rockford, Illinois, USA). Then, $30 \mu \mathrm{g}$ of total protein was separated via 10\% SDS-PAGE and transferred onto nitrocellulose membranes (Millipore, Temecula, California, USA). Next, the membrane was blocked and investigated with rabbit anti-human phospho-ERK1/2 antibody, rabbit anti-human ERK1/2 antibody, rabbit anti-human p-MEK1/2 antibody, rabbit anti-human MEK1/2 antibody, rabbit anti-human CLDN2 antibody, mouse anti-human $\beta$-actin antibody, and rabbit anti-human afadin antibody at a 1:1000 dilution at $4{ }^{\circ} \mathrm{C}$ for $12 \mathrm{~h}$. After three washes with phosphatebuffered saline (PBS), the membrane was incubated with horseradish peroxidase (HRP)-conjugated secondary antibody (Santa Cruz Biotechnologies, California, USA) at a 1:1000 dilution at room temperature (RT) for $30 \mathrm{~min}$. Immunoreactive bands were detected using ECL western blotting reagents (GE, Fairfield, Connecticut, USA) and analyzed with Image Lab 6.0.1 Software from Bio-Rad Laboratories.

\section{Immunofluorescence method}

Cells were washed thrice with phosphate-buffered saline (PBS), fixed with $4 \%$ paraformaldehyde for $10 \mathrm{~min}$ at RT, permeabilized with $0.1 \%$ Triton X-100 (SigmaAldrich; \#9002-93-1) for $10 \mathrm{~min}$ at RT and blocked in $2 \%$ bovine serum albumin (Bote Biotechnological Corporation, Shandong, China) in PBS for $1 \mathrm{~h}$ at RT. Staining with primary antibodies was performed with rabbit anti-human CLDN2 and rabbit anti-human afadin antibodies, which was diluted in blocking solution (1:1000 dilution) for $30 \mathrm{~min}$ at RT. The cells were incubated with Alexa Fluor ${ }^{\circledR} 647$-conjugated anti-rabbit IgG antibody (ab150093, Santa Cruz Biotechnologies, California, USA) at a 1:1000 dilution. Images were taken using an Olympus IX81 microscope with an MT20/20 illumination system.

\section{Immunoprecipitation}

Cells in the logarithmic growth phase were harvested and lysed in $600 \mu \mathrm{l}$ of pre-chilled protein lysis buffer $\left(4{ }^{\circ} \mathrm{C}\right)$ for $30 \mathrm{~min}$. The lysate was centrifuged at $14,000 \mathrm{rpm}$ at $4{ }^{\circ} \mathrm{C}$ for $20 \mathrm{~min}$, after which the protein concentration was determined with a Beyotime protein assay kit. The protein supernatant was divided into two volumes. One $50-\mu \mathrm{l}$ volume was mixed with $5 \times$ protein loading buffer, boiled in a water bath for $10 \mathrm{~min}$, and then was stored at $-20{ }^{\circ} \mathrm{C}$ as the input control. The remaining volume of lysate was mixed with $30 \mu \mathrm{l}$ of protein $\mathrm{A} / \mathrm{G}$ agarose beads and $2 \mu \mathrm{g}$ of a normal IgG antibody of the same species as the IgG used for immunoprecipitation. The mixture was rotated at $4{ }^{\circ} \mathrm{C}$ for $1 \mathrm{~h}$ and then centrifuged at $2500 \mathrm{rpm}$ at $4{ }^{\circ} \mathrm{C}$ for $5 \mathrm{~min}$, after which the supernatant was transferred to a new centrifuge tube. Next, $2 \mu \mathrm{g}$ of the primary immunoprecipitation antibody (rabbit anti-human raf-1 antibody) was added to the total protein, and the mixture was adjusted to a final volume of $600 \mu \mathrm{l}$ with protein lysis buffer. The mixture was slowly mixed overnight at $4{ }^{\circ} \mathrm{C}$ on a shaker. Forty microliters of adequately mixed protein A/G agarose beads was added to the mixture, which was slowly rotated at $4{ }^{\circ} \mathrm{C}$ for $90 \mathrm{~min}$ and then centrifuged at $3000 \mathrm{rpm}$ at $4{ }^{\circ} \mathrm{C}$ for $10 \mathrm{~min}$. Afterwards, the supernatant was removed, and the protein $\mathrm{A} / \mathrm{G}$ agarose pellet was saved. Protein lysis buffer was used to wash the protein A/G agarose pellet five times, after which $25 \mu \mathrm{l}$ of protein lysis buffer was added to resuspend the pellet followed by the addition of $5 \times$ loading buffer. The mixture was boiled for 5-10 min and used for sodium dodecyl sulfate-polyacrylamide gel electrophoresis (SDS-PAGE).

\section{Cell counting kit-8 assay}

A cell proliferation curve was determined by the colorimetric water-soluble tetrazolium salt assay (Cell counting kit-8; Dojindo, Kumamoto, Japan) according to the manufacturer's protocol. The cells were seeded into 96-well plates in triplicate, and cell proliferation was recorded every $12 \mathrm{~h}$ for 4 days.

\section{Colony formation assays}

The experimental method was the same as describe previously [21].

\section{Flow cytometry cell cycle analysis}

The experimental method was the same as describe previously [21].

\section{Wound-healing assay}

Cells were cultured in a monolayer at $70 \%$ confluence on gridded plastic dishes. Monolayers were wounded by scratching with a $100-\mu l$ pipette tip and subsequently washed 3 times with phosphate buffer saline. Images of the wound site were taken using a light microscope (E100, Nikon Instruments Inc, Japan) (magnification 200×) at the same location at $0,12 \mathrm{~h}$ and $24 \mathrm{~h}$, respectively.

\section{Transwell chamber method}

The cells were grown in a monolayer at $90 \%$ convergence and were maintained in FBS-free medium for $12 \mathrm{~h}$. Matrigel (BD Biosciences, cat. no. 356234) was added to the upper Boyden chamber (Millipore, Bedford, MA) in 24-well plates and the plates were maintained in a cell incubator at $37{ }^{\circ} \mathrm{C}$ for $15 \mathrm{~min}$. Then, medium containing 
chemotactic factors, which had been collected from the cell culture, was added to the 24-well plate. The cells were supplemented with Matrigel and cultured in a cell incubator at $37^{\circ} \mathrm{C}$ for $6 \mathrm{~h}$.

\section{Short hairpin RNA (shRNA) method}

Frozen glycerol bacterial stocks containing pGCSILscramble, pGCSIL-CLDN2-RNAi and pGCSIL-afadinRNAi frozen glycerol bacterial stocks were purchased from Nanjing KeyGen Biotech Co, Ltd. HEK 293T cells $\left(0.2 \times 10^{7}\right)$ were seeded and maintained for $24 \mathrm{~h}$ to achieve $70-80 \%$ confluence in 6-well dishes (Costar, Cam- bridge, MA). The plasmids, including $10 \mu \mathrm{g}$ pGCSIL-afadin-RNAi, pGCSIL-CLDN2-RNAi or pGCSILscramble, $5 \mu \mathrm{g}$ packaging vector pHelper 1.0 and $5 \mu \mathrm{g}$ vesicular stomatitis virus glycoprotein (VSVG) expression plasmid vector, were supplemented with Opti-MEM and $1.0 \mathrm{ml}$. A total of $50 \mu \mathrm{l}$ of Lipofectamine was added to $950 \mu \mathrm{l} \mathrm{FBS}$-free medium. These two mixtures were mixed and added to the cells. Lentiviral particles were harvested $48 \mathrm{~h}$ after transfection, and the viral titer was determined by counting green fluorescent protein (GFP)expressing cells under fluorescence microscopy (Nikon Diaphot $300^{\circledR}$ ) with filters $96 \mathrm{~h}$ after transfection.

\section{Patients and tissue samples}

Biopsies were gathered from 27 patients with a pathologically confirmed diagnosis of OS who received treatment at the Qilu Hospital of Shandong University between June 2007 and May 2012. The patients were chosen based on the following criteria: no history of radiotherapy, no history of chemotherapy, and no prior malignant disease. The grade and classification of OS patients were referred to the American Joint Committee on Cancer (AJCC) tumor node metastasis (TNM) staging system. 3 of the biopsies were taken from the tibia, 3 from the humerus, 16 from the femur, 2 from the fibula, and 1 each from the forearm, the hand, and the pelvis. 21 of the cases also exhibited pulmonary metastasis. Bone tissue that was identified as histologically non-neoplastic was also obtained from 10 patients with osteoarthritis who were treated at the Qilu Hospital of Shandong University between October 2006 and September 2011. There were 6 men and 4 women with an average age of 47 years.

\section{Immunohistochemistry}

Immunohistochemistry was used to detect the expression levels of CLDN2 and afadin in paraffin-embedded biopsy specimens from 27 patients who had a diagnosed primary OS and had not undergone chemotherapy or radiotherapy before biopsy. The experimental method was the same as described previously [21], and the antibodies used were rabbit anti-human CLDN2 and afadin antibody. Negative control slides were incubated with isotype antibodies at same dilution with primary antihuman CLDN2 and afadin antibody. The expression levels of CLDN2 and afadin located at the cell membrane and cytoplasm were taken as positive. The staining and scoring of the CLDN2 and afadin protein expression levels were classified semi-quantitatively based on the total combined scores of the percentage of positively stained tumor cells together with the staining intensity as previously described [22]. The final score of the protein expression was defined as 'low' if $<30 \%$ of tumor cells stained positive, and 'high' if $>30 \%$ of tumor cells stained positive. At least five different areas of the tumor were examined, and the mean of the results was used as the final expression score in each case.

\section{Statistical methods}

All of the experiments were repeated three times, and all of the data are based on the mean $\pm S D$ of at least three experimental results. The Chi-square test/Chi-square goodness of fit test was used to analyze the correlations between CLDN2 and afadin expression and clinical pathological indicators. The results were analyzed by Student's t-test, and $P<0.05$ was considered to be statistically significant.

\section{Results}

\section{Expressions of CLDNs family members in OS cell lines and osteoblasts}

Real-time quantitative PCR and western blotting were used to detect the expression of CLDNs family members in fetal-osteoblast cell line, hFOB.1.19 and OS cells U2OS, Saos2. The results showed that the mRNA and protein of CLDN1, CLDN3, CLDN4, CLDN5, CLDN6 and CLDN7 were not expressed in osteoblast cell lines and OS cell lines. Meanwhile, the mRNA and protein of CLDN8 was expressed in osteoblasts and OS cell lines, but there was no significant difference between the mRNA and protein expression of CLDN8 in osteoblasts and OS cell lines. Furthermore, the mRNA and protein of CLDN2 is expressed in low expression in OS cell lines and is high expressed in osteoblasts (Fig. 1a-c). It is suggested that the silence of CLDN2 expression may play a role in the occurrence of OS. Furthermore, the protein expression of afadin, a potential target gene of CLDN2, is also expressed in low expression in OS cell lines and is high expressed in osteoblasts.

Besides, CLDN2 expression was explored in 27 OS tissues and 10 histologically non-neoplastic bone tissues. As shown in Fig. 1d, the expression of CLDN2 in histologically non-neoplastic bone tissues and OS tissues is mainly located in the cytoplasm and membrane. High expression of CLDN2 was observed in 33.3\% (9/27) of 


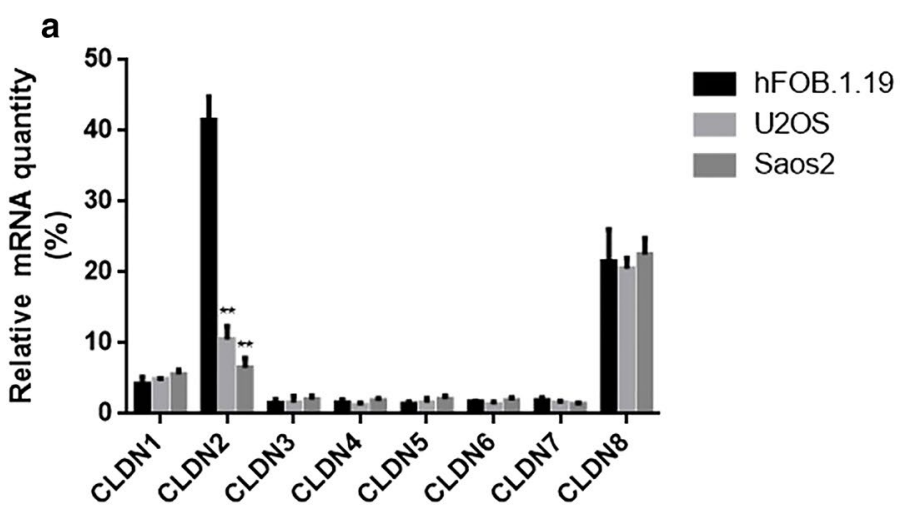

b

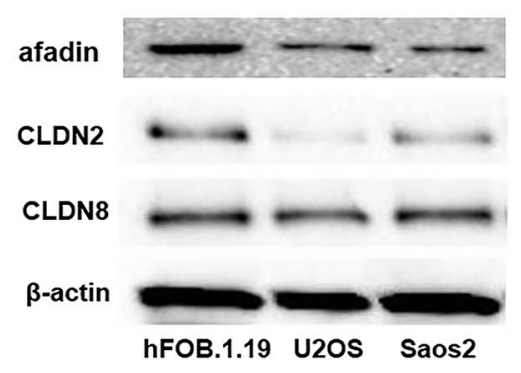

\section{C}

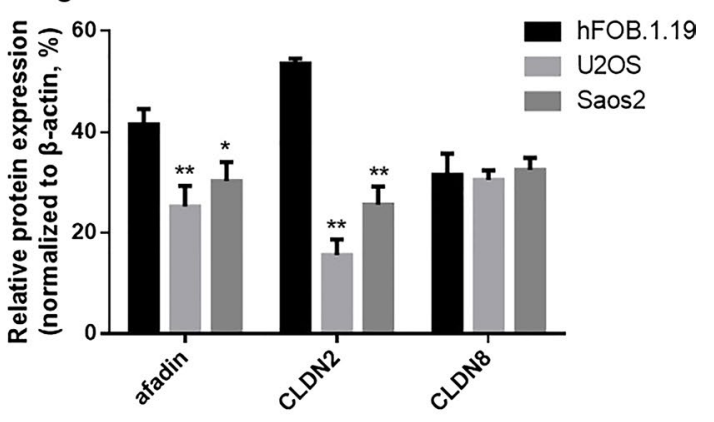

d

Normal tissue

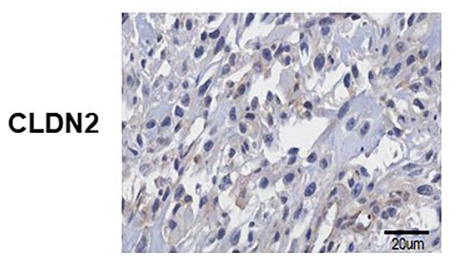

OS with no metastasis

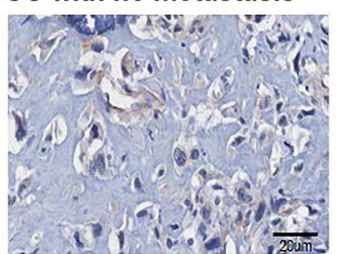

OS with metastasis

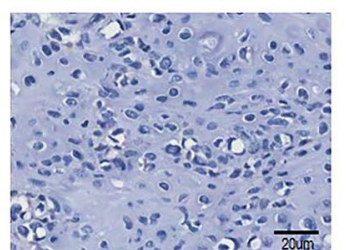

Fig. 1 Expressions of CLDNs family members in OS cell lines and osteoblast line. a Detection of the expression of CLDNs family members in OS cell lines and osteoblast line by Realtime-PCR. $\mathbf{b}$ Detection of CLDN2 expression in OS cell lines and osteoblast line by Western blotting. $\mathbf{c}$ The corresponding statistical analysis of protein expression. $\mathbf{d}$ The protein expression of CLDN2 in OS tissues and normal bone tissues. Normalized with $\beta$-actin, the ${ }^{*} P<0.05,{ }^{* *} \mathrm{P}<0.01$ compared with the hFOB.1.19 group

OS tissues and in $80.0 \%(8 / 10)$ of histologically non-neoplastic bone tissues (Table 1). These data revealed that the expression of CLDN2 was decreased in OS tissues compared with histologically non-neoplastic bone tissues $(\mathrm{P}=0.0001)$ (Table 1). The relationship between CLDN2 and clinical pathological indicators was also analyzed, and it was found that the expression of CLDN2 was not associated with age $(P=0.871)$, gender $(P=1.000)$, stage $(P=0.726)$, or response to chemotherapy $(P=0.642)$ of OS patients. However, CLDN2 expression was associated with pulmonary metastasis $(P=0.001)$ (Table 1$)$.

\section{Stable transfection of OS cell line U2OS with CLDN2}

In our presented work, CLDN2 was expressed at low level in Saos2 cells and undetectable in U2OS cells.
Therefore, to examine the consequence of an increase in CLDN2 expression, we stably over-expressed CLDN2 in U2OS cells. A pNSE-IRES-EGFP1-C1/CLDN2 plasmid was used to transfect U2OS cells. After G418 screening, a mixer with ten monoclonal strains of U2OS cells transfected with a pNSE-IRES-EGFP1C1/CLDN2 plasmid was obtained, which was termed U2OS-CLDN2. Western blotting and immunofluorescence were used to detect the expressions and localizations of CLDN2 in U2OS cells. The results showed that the protein expression levels of CLDN2 in the clonal U2OS cells were significantly higher $(P=0.0014)$ than those in the empty vector groups (Fig. 2a, b). Besides, the expression of CLDN2 was primarily localized on cell membrane and cytoplasm (Fig. 2c). The results demonstrated that U2OS cell line that stably expressed CLDN2 were successfully established. 


$\begin{aligned} & \text { Table } 1 \\ & \text { characteristics in }\end{aligned}$
OS patients

NA not available

* Statistical significance was found with the Chi-square test/Chi-square goodness-of-fit test

\section{The impact of CLDN2 on the proliferation rate} and metastasis ability of OS cell

The growth curve of U2OS cells was drawn by CCK- 8 method. As it revealed in Fig. 2d, the data revealed that there was no significant difference in the proliferation rate between U2OS-CLDN2 cells and empty vector groups. We also determined the abilities of CLDN2expressing cells to form colonies, in both 2D monolayer culture (Fig. 2e). there was no significant difference in the number of colonies formed between U2OS-CLDN2 cells and empty vector groups $(P=0.716)$. Moreover, we explored whether CLDN2 affect the cell cycle progression. The cell cycle distribution of U2OS cells was analyzed using flow cytometry. A representative histogram for each cell line is shown in Fig. 2f, g, and the percentage of cells with a $S$ phase DNA content is plotted. The results revealed no differences in the percentage of U2OS cells in $\mathrm{S}$ phase $(P=0.647)$, between cells lines with and without CLDN2 expression, suggesting that CLDN2 has no effect on cell cycle progression.

A wound-healing experiment was also used to detect the impact of CLDN2 on the migration ability of OS cells (Fig. 2h). The results showed that at $12 \mathrm{~h}$ and $24 \mathrm{~h}$, the migration distances of U2OS-CLDN2 $(P=0.036$; $P=0.0021)$ was significantly shorter than those of the empty vector groups. To determine the impact of CLDN2 on cell metastasis, the transwell chamber method was used to detect the invasive ability of OS cells. Six hours after the U2OS cells were seeded, those cells that invaded under the membrane of the chamber were observed (Fig. 2i). The results showed that the numbers of invasive U2OS-CLDN2 $(P=0.016)$ cells were significantly lower than those for the empty vector groups (Fig. 2j). This result suggests that CLDN2 significantly inhibits the invasive ability of U2OS cells in vitro.

These results suggest that CLDN2 significantly inhibits the migration ability of OS cells in vitro.

\section{The impact of CLDN2 on the ERK signaling pathway of OS cells}

Western blotting was used to detect the activation state of the Ras/Raf/MEK/ERK pathway. As it showed in Fig. 3a, b, after the overexpression of CLDN2, the ratio of phosphorylation ERK1/ERK1 $(P=0.0026)$ and phosphorylation ERK2/ERK2 $(P=0.0015)$ were significantly decreased in U2OS cells. Besides, the phosphorylation levels of MEK1/2 $(P=0.0023 ; P=0.0017)$, an upstream regulator of ERK1/2, were also significantly decreased in the U2OS cells that overexpressed CLDN2. In addition, upstream Ras activity in the U2OS $(P=0.024)$ cells were significantly decreased accompanied by the overexpression of CLDN2. However, the expression of afadin which is a potential target gene of CLDN2 was significantly increased in U2OS-CLDN2 group $(P=0.0012)$. These data suggest that CLDN2 significantly inhibits the activation of the Ras/Raf/MEK/ERK signaling pathway.

\section{(See figure on next page.)}

Fig. 2 The impact of CLDN2 on the proliferation and metastasis ability of U2OS cells in vitro. a Detection of CLDN2 expression in the U2OS cells by Western blotting. $\mathbf{b}$ The corresponding statistical analysis of protein expression. $\mathbf{c}$ Detection of CLDN2 expression in the U2OS cells by immunofluorescence. $\mathbf{d}$ The growth curve of the U2OS cells was drawn by the CCK-8 method. $\mathbf{e}$ The abilities of U2OS cells to form colonies were detect by colony formation assay. $\mathbf{f}$ The cell cycle distribution of U2OS cells was analyzed using flow cytometry. $\mathbf{g}$ The percentage of cells with $\mathbf{S}$ phase DNA content from U2OS cells. $\mathbf{h}$ The transwell chamber method was utilized to detect the invasive ability of the U2OS cell line in vitro. $\mathbf{i}$ The wound healing assay was utilized to detect the migration ability of the U2OS cell line in vitro. ${ }^{*} P<0.05,{ }^{* *} \mathrm{P}<0.01$, compared with the control group compared with the vector group 
a

C
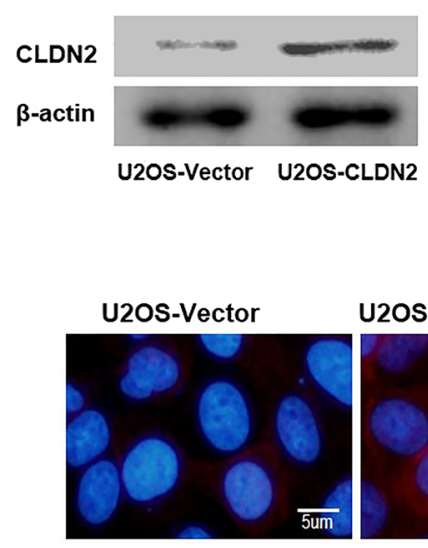

d

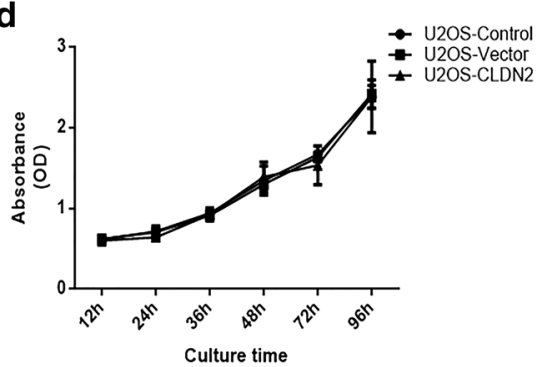

f

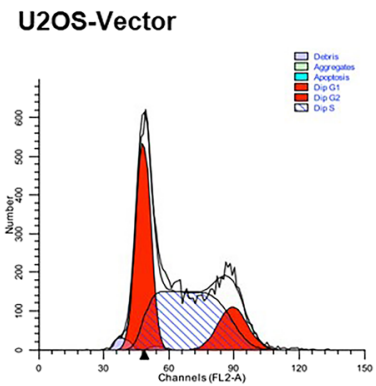

g

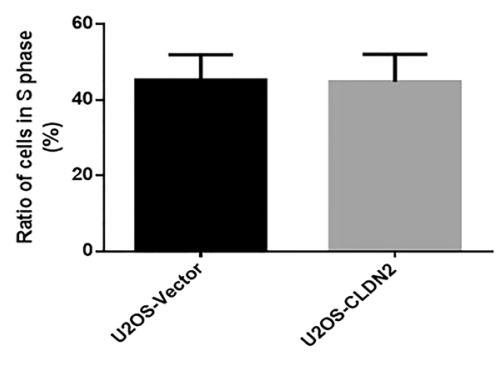

i U2OS-Vector

U2OS-CLDN2
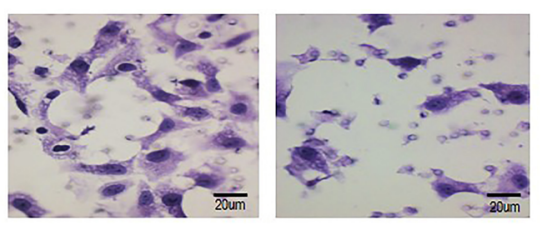

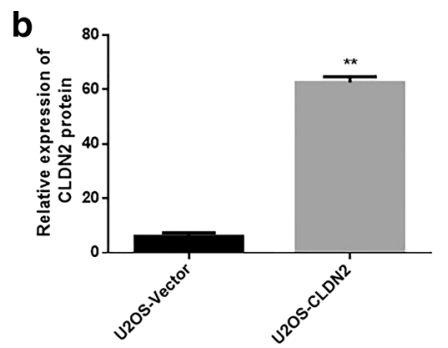

OS-CLDN2
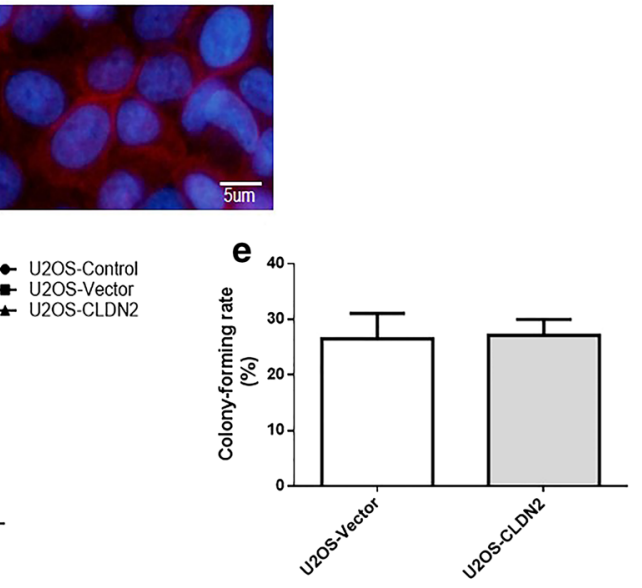

U2OS-CLDN2

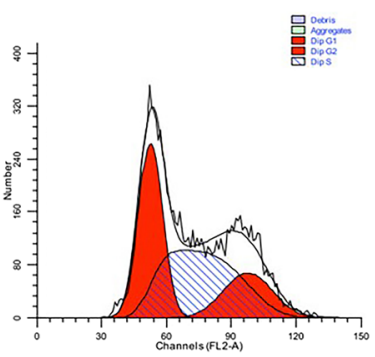

h

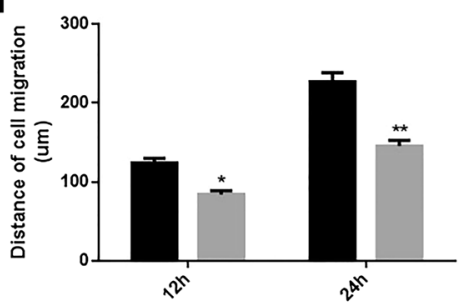

j

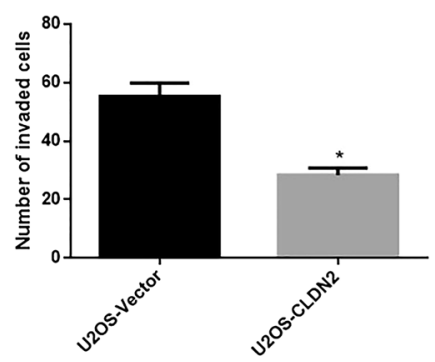



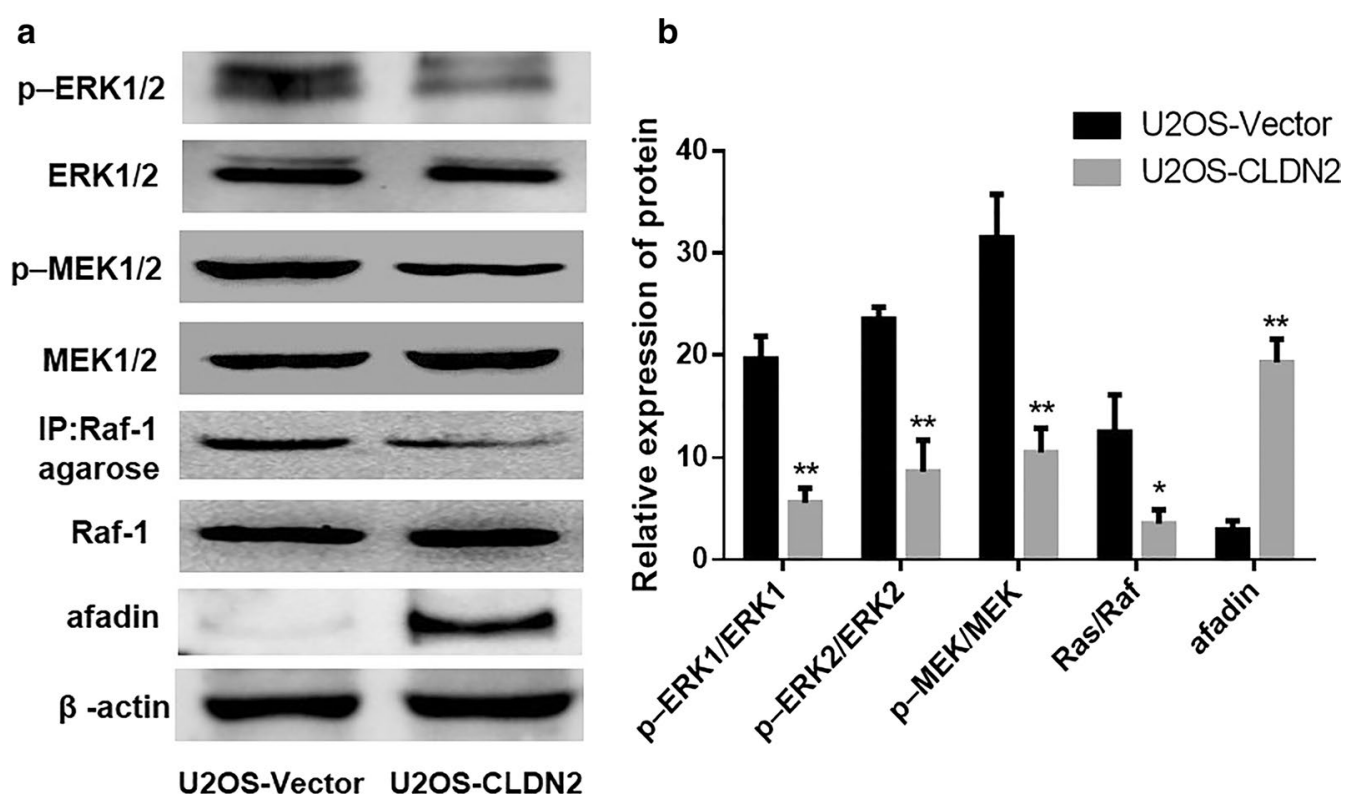

Fig. 3 The impact of CLDN2 on the Ras/Raf/MEK/ERK signaling pathway of U2OS cells. a Western blotting was used to detect the activation of the Ras/Raf/MEK/ERK signaling pathway in U2OS cells. b The corresponding statistical analysis of the activation of the Ras/Raf/MEK/ERK signaling pathway. ${ }^{*} P<0.05,{ }^{* *} \mathrm{P}<0.01$ compared with the control group, compared with the vector group

The impact of CLDN2 knockdown on the metastasis of fetal-osteoblast cell line

To determine the impact of CLDN2 on invasion and migration ability of osteoblast cell, we transfected fetalosteoblast cell line, hFOB.1.19 with a pGCSIL-scramble plasmid and a pGCSIL-CLDN2-RNAi plasmid. The western blotting was used to analyze the expression of CLDN2 and changes in the activation state of Ras/Raf/ MEK/ERK pathway in these cells, as it showed in Fig. 4a, $\mathrm{b}$, the ratio of phosphorylation ERK1/2 $(P=0.0013$; $P=0.0027)$ and phosphorylation MEK $1 / 2 \quad(P=0.0026$; $P=0.0003)$ were significantly increased in the hFOB.1.19 cells that silence CLDN2. In addition, upstream Ras activity in the hFOB.1.19 $(P=0.017)$ cells were significantly increased accompanied by the silence of CLDN2. However, the expression of afadin was significantly decreased in the hFOB.1.19 cells that silence CLDN2 $(P=0.0042)$.

The transwell chamber assay and wound-healing assay were used to analyze the effect of CLDN2 on the invasive and migration ability of the examined cells. The results showed that the migration distances of HFOB.1.19 cells in the CLDN2-RNAi group were significantly longer than those of the scramble group at $12 \mathrm{~h}$ and $24 \mathrm{~h}(P=0.0008$; $P=0.0001$ ) (Fig. 4c). The numbers of invasive HFOB.1.19 cells in the CLDN2-RNAi group $(P=0.004)$ were significantly increased following CLDN2 silencing (Fig. 4d, e).
Impact of silencing of afadin on Ras/Raf/MEK/ERK pathway activation

To determine whether CLDN2 affects the ERK signaling pathway by regulating afadin expression, a pGCSILscramble plasmid and a pGCSIL-afadin-RNAi plasmid were used to transfect CLDN2-expressing U2OS cells. Western blotting was used to analyze the effects of silencing afadin in these cells, and the results showed that compared with the control group, the expression of afadin was significantly reduced in afadin-RNAi group (Fig. 5a). Western blotting was used to examine changes in the activation state of ERK pathway. After afadin silencing, the phosphorylation levels of MEK1/2 $(P=0.0021$; $P=0.0001)$ and ERK1/2 $(P=0.0023 ; P=0.0011)$ were significantly increased in CLDN2-expressing U2OS cells (Fig. 5a, b). Besides, the loss of afadin have no notably impact on the CLDN2 expression $(P=0.796)$.

Futhermore, a transwell chamber assay and a woundhealing were used to analyze the effect of afadin on the metastasis ability of the examined cells. The results showed the migration distances of CLDN2-expressing cells at $12 \mathrm{~h}$ and $24 \mathrm{~h}$ were significantly increased following afadin silencing $(P=0.0021 ; P=0.0013)$ (Fig. 5c). In addition, the numbers of invasive cells in the CLDN2expressing cells were significantly increased following afadin silencing (Fig. 5d, e). 
a

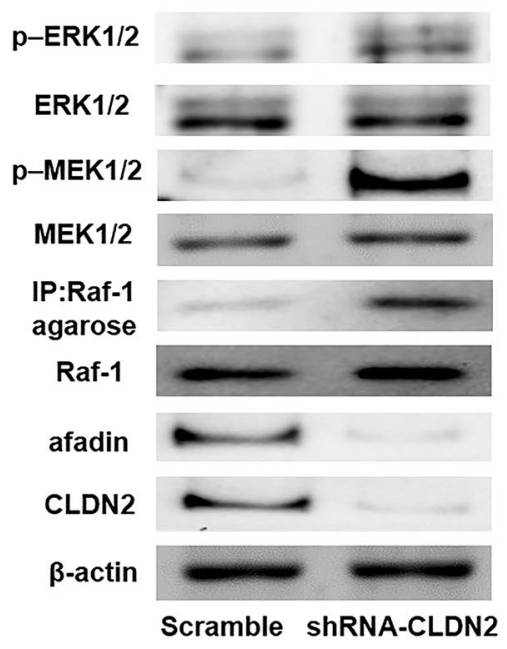

d

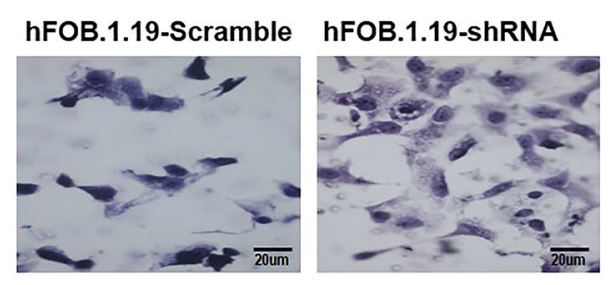

b

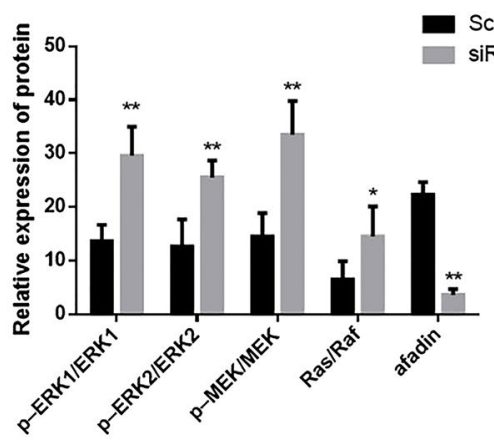

Scramble

SIRNA-CLDN2

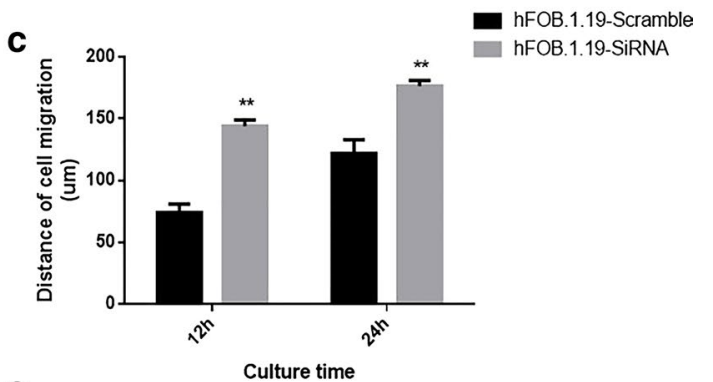

e

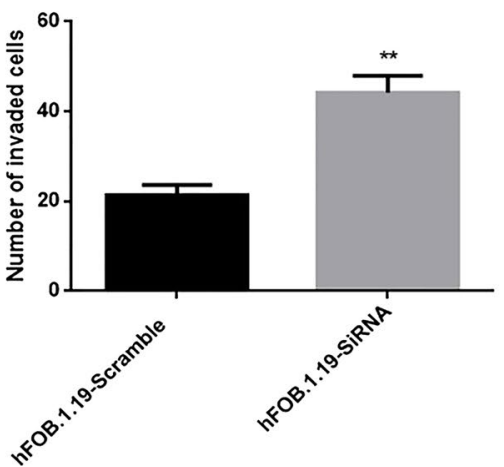

Fig. 4 RNAi was utilized to silence CLDN2 expression in osteoblast line. a Western blotting was utilized to examine the effects of silencing CLDN2 and the activation of the Ras/Raf/MEK/ERK signaling pathway in the osteoblast line. $\mathbf{b}$ The corresponding statistical analysis of the activation of the Ras/Raf/MEK/ERK signaling pathway. c The wound healing assay was utilized to detect the migration ability of the osteoblast line in vitro. $\mathbf{d}$ The transwell chamber method was utilized to detect the impact of CLDN2 silencing on the invasive ability of cells in vitro. e The corresponding statistical analysis of invaded cells. ${ }^{* *} P<0.01$ compared with the scramble group

\section{Expressions of afadin in human OS}

The expression of afadin was explored in the 27 specimens containing OS tissues and 10 specimens containing non-neoplastic bone tissue. The expressions of afadin located at cell membrane and cytoplasm were taken as positive. As shown in Fig. 5f, the expressions of afadin in OS tissues were remarkably lower than in non-neoplastic bone tissues $(\mathrm{P}=0.0005)$.

The relationship among afadin expression and clinical pathological indicators was analyzed, and it was found that the expression of afadin was not associated with the age $(P=0.341)$, gender $(P=1.000)$, clinical staging $(P=0.584)$ or response to chemotherapy $(P=0.127)$ but associated with pulmonary metastasis $(P=0.0003)$ in OS patients (Table 2). Besides, Chi-square/Chisquare goodness of fit test showed that afadin was positively correlated with CLDN2 expression $(\phi=0.793$, $P=0.011$ ) (Table 3).

\section{Discussion}

Recent research has revealed that the expression of tight junction protein CLDNs is frequently altered in various cancers [23]. CLDN2 is one of the 27 members of the CLDN protein family, and the current understanding of the biological functions of CLDN2 is primarily limited to barrier protection, and cell connections [24]. Our research group found that CLDN2 was underexpressed in OS tissues, and we hypothesized that this decrease in 

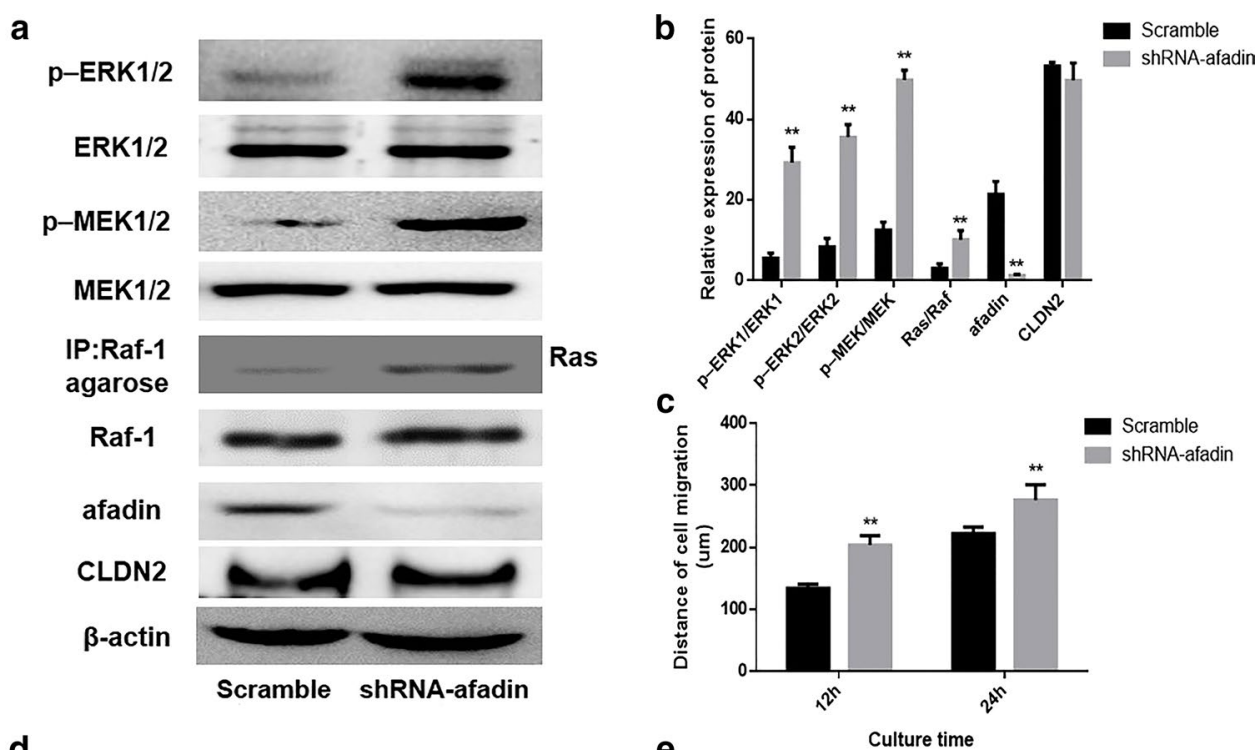

d
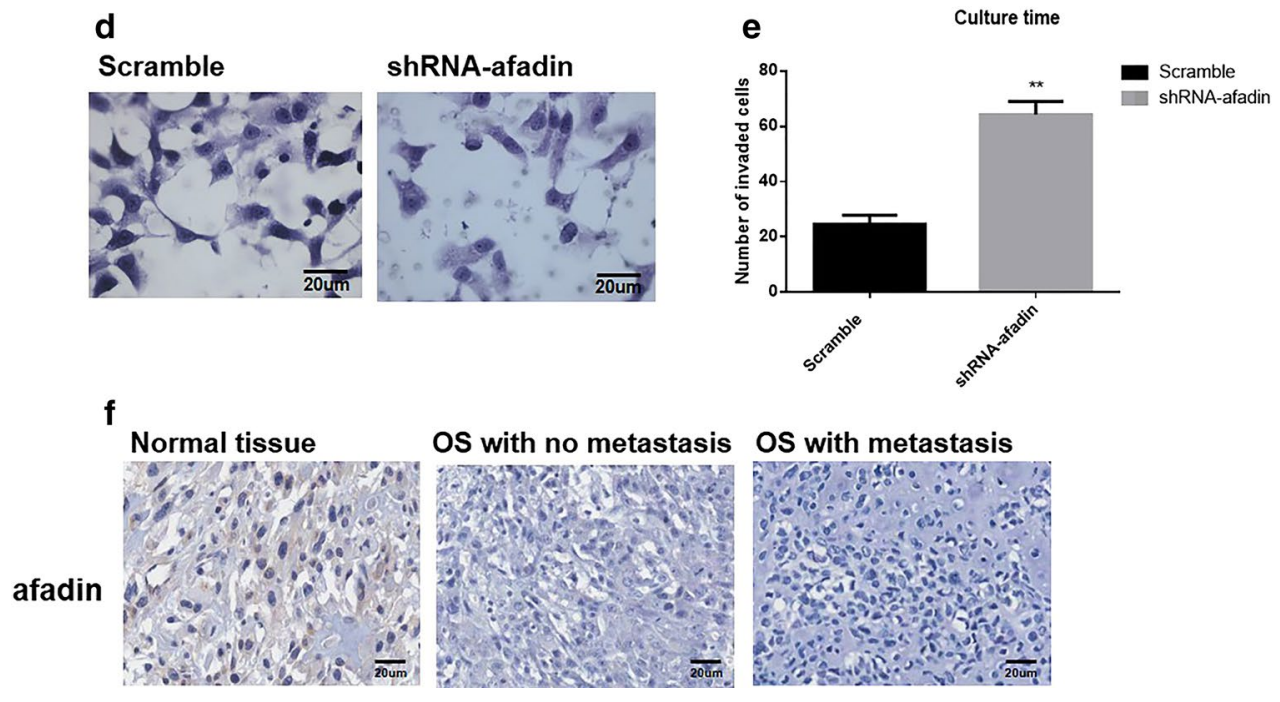

Fig. 5 Impact of afadin silencing on the metastasis phenotype of U2OS cells. a Western blotting was used to examine the effects of silencing afadin and the activation of the Ras/Raf/MEK/ERK signaling pathway in the U2OS cell line. $\mathbf{b}$ The corresponding statistical analysis of the activation of the Ras/Raf/MEK/ERK signaling pathway. c The wound healing assay was utilized to detect the migration ability of the U2OS cell line in vitro. $\mathbf{d}$ The transwell chamber method was utilized to detect the impact of afadin silencing on the invasive ability of cells in vitro. e The corresponding statistical analysis of invaded cells. $\mathbf{f}$ Membrane expressions of afadin in OS tissues and normal bone tissues. ${ }^{*} \mathrm{P}<0.01$ compared with the scramble group

gene expression may play a role in the metastasis phenotype of OS. To verify this hypothesis, we created an OS cell line stably expressing CLDN2 and an osteoblast cell line with a CLDN2 knockout. It is indicated that overexpression of CLDN2 significantly inhibited the metastasis and migration abilities of OS cells. Similar to our study, recent studies demonstrated that the CLDNs was frequently down-regulated in various cancers, for instance, the expression of CLDN1 was down-regulated in pancreatic cancer cells and that re-expression of CLDN1 reduced the invasive ability of these cells $[23,25]$. By contrast, others have reported that the expression of certain CLDNs in tumors is associated with strong invasion and metastasis abilities $[10,26]$. Thus, the various CLDNs may have different impacts on the biological behavior of a certain tumor [27-29]. One potential reason for this difference is that CLDNs may have specific functions in different cells and rely on different interacting molecules [30, 31]. For instance, CLDN1 was reported to induce cell migration and invasion through activation of the c-AblERK signaling pathway in human liver cells [32]. Parallelly, it is revealed that CLDN18 coupled with EGFR/ERK 
Table 2 Expression of afadin and clinicopathological characteristics in OS patients

\begin{tabular}{|c|c|c|c|c|}
\hline Item & $\mathrm{N}$ & Afadin (high) & Afadin (low) & $P$ \\
\hline Tumor tissue & 27 & 11 & 16 & $<0.001^{*}$ \\
\hline Normal & 10 & 8 & 2 & \\
\hline \multicolumn{5}{|l|}{ Age (years) } \\
\hline$\leq 19$ & 15 & 6 & 9 & \multirow[t]{2}{*}{0.341} \\
\hline$>19$ & 12 & 5 & 7 & \\
\hline \multicolumn{5}{|l|}{ Gender } \\
\hline Male & 16 & 6 & 10 & \multirow[t]{2}{*}{1.000} \\
\hline Female & 11 & 5 & 6 & \\
\hline \multicolumn{5}{|l|}{ Stage } \\
\hline$|A-| \mid A$ & 10 & 4 & 6 & \multirow[t]{2}{*}{0.584} \\
\hline$\|B-\| \mid$ & 17 & 7 & 10 & \\
\hline \multicolumn{5}{|c|}{ Response to chemotherapy } \\
\hline Poor & 9 & 3 & 6 & \multirow[t]{3}{*}{0.127} \\
\hline Good & 7 & 1 & 6 & \\
\hline$N A(n=8)$ & 11 & & & \\
\hline \multicolumn{5}{|c|}{ Pulmonary metastasis } \\
\hline+ & 21 & 7 & 14 & \multirow[t]{2}{*}{$<0.001$} \\
\hline- & 6 & 4 & 2 & \\
\hline
\end{tabular}

NA not available

* Statistical significance was found with the Chi-square test/Chi-square goodness-of-fit test

Table 3 Correlation between the expression of afadin and CLDN2 in OS tissues

\begin{tabular}{llll}
\hline Item & CLDN2 (high) & CLDN2 (low) & $P$ \\
\hline $\mathrm{n}$ & 9 & 18 & \\
Afadin (high) & 6 & 5 & $<0.01^{*}$ \\
Afadin (low) & 3 & 13 & \\
\hline
\end{tabular}

${ }^{*} \varphi$ Phi coefficient

signaling contributes to the malignant potential of bile duct cancer [33]. However, there have been few reports on the roles of CLDNs in OS, and the specific molecular mechanisms remain to be clarified.

Latest studies have shown that the cytoplasmic C-terminus of CLDNs contains a PDZ-binding sequence, which binds other tight junction proteins on the cytoplasmic side of cell membranes [34]. These intracellular proteins participate in cellular signal transduction and thereby regulate a series of cell behaviors [35]. In present study, afadin, which contains a PDZ domain ligand [3639], was identified as a potential target gene of CLDN2 protein.

Our data showed that afadin silencing results in a reactivation of the ERK signaling pathway and promotes the metastasis phenotype in OS cells stably expressing CLDN2. Moreover, our data suggested that the expression levels of both CLDN2 and afadin are found to be associated with pulmonary metastasis in OS tissues, suggesting that reduced CLDN2 and afadin expression is likely to participate in the pulmonary metastasis of OS. Hence, the expression levels of afadin and CLDN2 have the potential to be useful as molecular markers for the diagnosis of OS as well as for the determination of prognosis.

\section{Conclusions}

In the present study, we confirmed that CLDN2 significantly inhibits the migration ability of U2OS cells. We also performed an initial exploration of the molecular mechanism of this effect, finding that CLDN2 affected the Ras/Raf/MEK/ERK signaling pathway via afadin and ultimately decreased the migration ability of OS cells.

\section{Abbreviations \\ CLDNs: claudins; ERK: extracellular signal-regulated kinase; DMSO: dimethyl sulfoxide; MEK1/2: mitogen-activated protein kinase kinase 1/2; Ras: rat sarcoma; Raf: rapidly accelerated fibrosarcoma; TACC3: transforming acidic coiled-coil protein 3; TJs: tight junctions; SDS-PAGE: sodium dodecyl sulfate- polyacrylamide gel electrophoresis; ZOs: zonula occludens.}

\section{Authors' contributions}

XZ performed the experiments and analyzed the data. HW and QL contributed to the conception and design of the study. TL revised the manuscript critically for important intellectual content. All authors read and approved the final manuscript.

\section{Author details}

${ }^{1}$ Center for Translational Medicine, Central Hospital of Zibo, Affiliated with Shandong University, Gong gingtuan Road 54Hao, Zibo, Shandong, China. ${ }^{2}$ Department of General Surgery, People's Hospital of Linzi District, Affiliated with Binzhou Medical College, Zibo, Shandong, China. ${ }^{3}$ Department of Orthopedic Surgery, Central Hospital of Zibo, Affiliated with Shandong University, Zibo, Shandong, China.

\section{Acknowledgements}

We would like to thank American Journal Experts (AJE) for English language editing.

\section{Competing interests}

The authors declare that they have no competing interests.

\section{Availability of data and materials}

The datasets used and/or analyzed during the present study are available from the corresponding author on reasonable request.

\section{Consent for publication}

All the listed authors have participated actively in the study, and have seen and approved the submitted manuscript.

\section{Ethics approval and consent to participate}

All procedures performed in studies involving human participants were in accordance with the ethical standards of the institutional and/or national research committee and with the 1964 Helsinki declaration and its later amendments or comparable ethical standards. Ethics approval (No. JLU12454) was approved by the Ethics Committee of Shandong University. This article does not contain any studies with animals performed by any of the authors.

\section{Funding}

This study was supported by National Natural Science Foundation of Shandong Province (Code: ZR2014HM042). 


\section{Publisher's Note}

Springer Nature remains neutral with regard to jurisdictional claims in published maps and institutional affiliations.

Received: 28 July 2018 Accepted: 10 October 2018 Published online: 17 October 2018

\section{References}

1. Salunke AA, Shah J, Gupta N, Pandit J. Pathologic fracture in osteosarcoma: association with poorer overall survival. Eur J Surg Oncol. 2016;42:889-90.

2. Anderson ME. Update on survival in osteosarcoma. Orthop Clin N Am. 2016:47:283-92.

3. Li X, Ashana AO, Moretti VM, Lackman RD. The relation of tumour necrosis and survival in patients with osteosarcoma. Int Orthop. 2011;35:1847-53.

4. Escudero-Esparza A, Jiang WG, Martin TA. The claudin family and its role in cancer and metastasis. Front Biosci. 2010;16:1069-83.

5. Cunniffe C, Brankin B, Lambkin H, Ryan F. The role of claudin-1 and claudin-7 in cervical tumorigenesis. Anticancer Res. 2014;34:2851-7.

6. Balda MS, Matter K. Tight junctions at a glance. J Cell Sci. 2008:121:3677-82.

7. González-Mariscal L, Tapia R, Chamorro D. Crosstalk of tight junction components with signaling pathways. Biochimica et Biophysica Acta (BBA) Biomembranes. 2008;1778:729-56.

8. Tsukita S, Furuse M, Itoh M. Structural and signalling molecules come together at tight junctions. Curr Opin Cell Biol. 1999;11:628-33.

9. Oliveira S, Morgado-Diaz J. Claudins: multifunctional players in epithelia tight junctions and their role in cancer. Cell Mol Life Sci. 2007:64:17-28.

10. Ouban A, Ahmed AA. Claudins in human cancer: A review. Histol Histopathol. 2010:25:83-90.

11. Tabaries S, Siegel PM. The role of claudins in cancer metastasis. Oncogene. 2017:36:1176-90.

12. Chao Y-C, Pan S-H, Yang S-C, Yu S-L, Che T-F, Lin C-W, Tsai M-S, Chang G-C, Wu C-H, Wu Y-Y. Claudin-1 is a metastasis suppressor and correlates with clinical outcome in lung adenocarcinoma. Am J Respir Crit Care Med. 2009; 179:123-33.

13. Usami Y, Chiba H, Nakayama F, Ueda J, Matsuda Y, Sawada N, Komori T, Ito A, Yokozaki H. Reduced expression of claudin-7 correlates with invasion and metastasis in squamous cell carcinoma of the esophagus. Hum Pathol. 2006:37:569-77.

14. Merino-Gracia J, Costas-Insua C, Canales MA, Rodriguez-Crespo I. Insights into the C-terminal peptide binding specificity of the PDZ domain of neuronal nitric-oxide synthase: characterization of the interaction with the tight junction protein claudin-3. J Biol Chem. 2016;291:11581-95.

15. Jeansonne B, Lu Q, Goodenough DA, Chen YH. Claudin-8 interacts with multi-PDZ domain protein 1 (MUPP1) and reduces paracellular conductance in epithelial cells. Cell Mol Biol. 2003;49:13-21.

16. Hamazaki Y, Itoh M, Sasaki H, Furuse M, Tsukita S. Multi-PDZ domain protein 1 (MUPP1) is concentrated at tight junctions through its possible interaction with claudin-1 and junctional adhesion molecule. J Biol Chem. 2002;277:455-61

17. Osanai M, Takasawa A, Murata M, Sawada N. Claudins in cancer: bench to bedside. Pflugers Arch. 2017;469:55-67.

18. Carmena A, Speicher S, Baylies M. The PDZ protein Canoe/AF-6 links RasMAPK, Notch and Wingless/Wnt signaling pathways by directly interacting with Ras. Notch and Dishevelled. PLoS ONE. 2006;1:e66.

19. Boettner B, Govek E-E, Cross J, Van Aelst L. The junctional multidomain protein AF- 6 is a binding partner of the Rap1A GTPase and associates with the actin cytoskeletal regulator profilin. Proc Natl Acad Sci. 2000;97:9064-9

20. Livak KJ, Schmittgen TD. Analysis of relative gene expression data using real-time quantitative $P C R$ and the $2(-\triangle \Delta C(T))$ method. Methods. 2001:25:402-8

21. Zhang X, Ruan Y, Li Y, Lin D, Quan C. Tight junction protein claudin-6 inhibits growth and induces the apoptosis of cervical carcinoma cells in vitro and in vivo. Med Oncol. 2015;32:148.

22. Yang Y, Yang H, McNutt MA, Xiong F, Nie X, Li L, Zhou R. LAPTM4B overexpression is an independent prognostic marker in ovarian carcinoma. Oncol Rep. 2008;20:1077-83.
23. Hewitt KJ, Agarwal R, Morin PJ. The claudin gene family: expression in normal and neoplastic tissues. BMC Cancer. 2006:6:186.

24. Koval M. Claudin heterogeneity and control of lung tight junctions. Annu Rev Physiol. 2013;75:551-67.

25. Morin PJ. Claudin proteins in human cancer: promising new targets for diagnosis and therapy. Can Res. 2005;65:9603-6.

26. Zavala-Zendejas VE, Torres-Martinez AC, Salas-Morales B, Fortoul TI, Montano LF, Rendon-Huerta EP. Claudin-6, 7, or 9 overexpression in the human gastric adenocarcinoma cell line AGS increases its invasiveness, migration, and proliferation rate. Cancer Investig. 2011;29:1-11.

27. D'Souza T, Agarwal R, Morin PJ. Phosphorylation of claudin-3 at threonine 192 by CAMP-dependent protein kinase regulates tight junction barrier function in ovarian cancer cells. J Biol Chem. 2005;280:26233-40.

28. D'Souza T, Indig FE, Morin PJ. Phosphorylation of claudin-4 by PKC $\varepsilon$ regulates tight junction barrier function in ovarian cancer cells. Exp Cell Res. 2007:313:3364-75

29. Li X, Li Y, Qiu H, Wang Y. Downregulation of claudin-7 potentiates cellular proliferation and invasion in endometrial cancer. Oncol Lett. 2013;6:101-5

30. Lu Z. Functions of claudin-7 in human lung cancer. 2012.

31. Micke P, Mattsson JS, Edlund K, Lohr M, Jirstrom K, Berglund A, Botling J, Rahnenfuehrer J, Marincevic M, Ponten F, et al. Aberrantly activated claudin 6 and 18.2 as potential therapy targets in non-small-cell lung cancer. Int J Cancer. 2014;135:2206-14

32. Suh Y, Yoon CH, Kim RK, Lim EJ, Oh YS, Hwang SG, An S, Yoon G, Gye MC, Yi JM, et al. Claudin-1 induces epithelial-mesenchymal transition through activation of the c-Abl-ERK signaling pathway in human liver cells. Oncogene. 2017;36:1167-8.

33. Takasawa K, Takasawa A, Osanai M, Aoyama T, Ono Y, Kono T, Hirohashi Y, Murata M, Sawada N. Claudin-18 coupled with EGFR/ERK signaling contributes to the malignant potentials of bile duct cancer. Cancer Lett. 2017;403:66-73.

34. Itoh M, Furuse $M$, Morita $K$, Kubota $K$, Saitou M, Tsukita S. Direct binding of three tight junction-associated MAGUKs, ZO-1, ZO-2, and ZO-3, with the $\mathrm{COOH}$ termini of claudins. J Cell Biol. 1999;147:1351-63.

35. Kominsky SL. Claudins: emerging targets for cancer therapy. Expert Rev Mol Med. 2006:8:1-11.

36. Fujiwara Y, Goda N, Tamashiro T, Narita H, Satomura K, Tenno T, Nakagawa A, Oda M, Suzuki M, Sakisaka T, et al. Crystal structure of afadin PDZ domain-nectin-3 complex shows the structural plasticity of the ligandbinding site. Protein Sci. 2015;24:376-85

37. Monteiro AC, Sumagin R, Rankin CR, Leoni G, Mina MJ, Reiter DM, Stehle T, Dermody TS, Schaefer SA, Hall RA, et al. JAM-A associates with ZO-2, afadin, and PDZ-GEF1 to activate Rap2c and regulate epithelial barrier function. Mol Biol Cell. 2013;24:2849-60.

38. Severson EA, Lee WY, Capaldo CT, Nusrat A, Parkos CA. Junctional adhesion molecule A interacts with Afadin and PDZ-GEF2 to activate Rap1A regulate beta1 integrin levels, and enhance cell migration. Mol Biol Cell. 2009:20:1916-25

39. Mandai $K$, Nakanishi $H$, Satoh A, Obaishi H, Wada M, Nishioka H, Itoh M, Mizoguchi A, Aoki T, Fujimoto T, et al. Afadin: a novel actin filament-binding protein with one PDZ domain localized at cadherin-based cell-to-cell adherens junction. J Cell Biol. 1997;139:517-28.

Ready to submit your research? Choose BMC and benefit from

- fast, convenient online submission

- thorough peer review by experienced researchers in your field

- rapid publication on acceptance

- support for research data, including large and complex data types

- gold Open Access which fosters wider collaboration and increased citations

- maximum visibility for your research: over 100M website views per year

At BMC, research is always in progress.

Learn more biomedcentral.com/submissions 